\title{
Linx
}

Revue des linguistes de l'université Paris X Nanterre

$12 \mid 2002$

"Comme la lettre dit la vie »

\section{Texte argumentatif et structures syntaxiques en Moyen Français : la topicalisation d'une proposition}

\section{Bernard Combettes}

\section{OpenEdition}

\section{Journals}

Édition électronique

URL : http://journals.openedition.org/linx/1278

DOI : 10.4000/linx.1278

ISSN : 2118-9692

Éditeur

Presses universitaires de Paris Nanterre

\section{Édition imprimée}

Date de publication : 1 octobre 2002

Pagination : 56-63

ISSN : 0246-8743

\section{Référence électronique}

Bernard Combettes, «Texte argumentatif et structures syntaxiques en Moyen Français : la topicalisation d'une proposition », Linx [En ligne], 12 | 2002, mis en ligne le 10 octobre 2012, consulté le 19 avril 2019. URL : http://journals.openedition.org/linx/1278; DOI : 10.4000/linx.1278

Ce document a été généré automatiquement le 19 avril 2019

Département de Sciences du langage, Université Paris Ouest 


\title{
Texte argumentatif et structures syntaxiques en Moyen Français : la topicalisation d'une proposition
}

\author{
Bernard Combettes
}

1 Les études diachroniques portant sur l'ordre des constituants ne peuvent faire l'économie, surtout lorsqu'il s'agit de l'analyse des constituants majeurs de la proposition, de notions qui relèvent du domaine de la thématisation et de celui de la topicalisation. L'évolution de la structure de la phrase en français ne parait pas pouvoir être interprétée de façon pertinente si l'on ne prend pas en compte la question des relations qui s'établissent entre les fonctions syntaxiques et les catégories de thème ou de topique. Même dans les travaux qui ne s'intéressent pas aux aspects textuels et discursifs, s'en tenant uniquement au niveau morphosyntaxique, on peut constater que les notions appartenant au domaine de la perspective fonctionnelle, ou, plus généralement, à la structure informationnelle de l'énoncé, sont considérées comme des unités dont le jeu est important et pertinent parmi les facteurs de changement. La confusion relative qui est parfois perceptible dans ce niveau d'analyse, ne serait-ce qu'en ce qui concerne les principaux concepts et les critères mis en œuvre pour les définir, ne doit pas faire abandonner ce champ de recherche. Il nous semble en particulier indispensable de distinguer le plus clairement possible les deux opérations fondamentales : thématisation et topicalisation. L'opposition thème / rhème peut ainsi être réservée à l'analyse du "dynamisme communicatif», avec toute la gradation qui permet de rendre compte de diverses sous-parties à l'intérieur de chacune des deux grandes catégories. Le début d'énoncé n'est évidemment pas la seule zone concernée par cette problématique, l'ordre relatif des constituants placés en fin de phrase jouant un grand rôle dans la délimitation $\mathrm{du}$ rhème propre, du moins dans une langue comme le français. La topicalisation introduit une dimension supplémentaire : la plupart des définitions s'accordent en effet pour voir dans le topique non seulement le «point de départ » de l'énoncé, mais le support d'une prédication, le constituant renvoyant au référent «au sujet duquel » est construit un commentaire. Cette différence peut être mise en parallèle avec les deux types de présupposés que Lambrecht (2001: 475-476) propose de distinguer lorsqu'il 
s'agit de rendre compte de la présentation des référents: une présupposition de " conscience» (le locuteur suppose que la représentation mentale du référent a été activée dans la mémoire à court terme de l'interlocuteur au moment de l'énonciation), une présupposition de topicalité (le locuteur suppose que le référent est considéré par le récepteur comme un "center of current interest » pouvant ainsi être le support d'une prédication).

2 La différence entre les deux opérations, qui entretiennent des relations étroites, dans la mesure où le topique pourrait être considéré comme un type particulier de thème, apparait dans l'opposition que l'on perçoit entre d'une part :

- Dans la conclusion apparaissent quelques bonnes remarques

- Dans la conclusion, il y a quelques bonnes remarques,

énoncés qui illustrent des cas de thématisation, avec une linéarisation qui pourrait être comparée à: -Quelques bonnes remarques apparaissent dans la conclusion. Il y a, dans la conclusion, quelques bonnes remarques, etc., le groupe initial ouvrant un cadre discursif mais n'étant pas lui-même l'objet de la prédication, et d'autre part :

- Quant à la conclusion, elle contient quelques bonnes remarques

- La conclusion, elle contient quelques bonnes remarques,

où la position périphérique du constituant initial ne code pas seulement le degré élevé d'activation du référent, mais aussi le fait que cette unité peut correspondre à un centre d'intérêt à partir duquel va se développer une prédication.

La distinction que nous venons rapidement d'évoquer peut être mise en rapport avec la typologie des textes et des discours. Sans qu'il soit évidemment envisageable d'établir des règles strictes de correspondance, on peut considérer que les opérations de topicalisation surviennent essentiellement dans les textes de type argumentatif et de type explicatif, alors qu'elles apparaissent moins souvent dans les textes narratifs, du moins dans des textes correspondant à des états de langue anciens. Les enchaînements d'énoncés s'y opèrent en effet dans une cohérence "resserrée ", étroite, qui, par l'intermédiaire de progressions thématiques linéaires, joue sur des reprises immédiates ou sur l'emploi de circonstants «cadres textuels ", temporels ou spatiaux (cf. Rychner, 1970; Combettes, 1988, 1992 ). Il est facile de constater, dans les textes narratifs en prose, la grande fréquence de liaisons interphrastiques du type suivant :

- en l'orée d'une grant forest (...) trouva un pavillon tendu, riche à merveilles (..). A

l'encontre duquel pavillon avoit une colombe de pierre de jaspe (...). En laquelle colombe $y$

avoit anciennes lettres artificiellement entaillées (...) (René d'Anjou, 1457),

enchaînements qui correspondent à une progression thématique linéaire. Dans un tel système, la présence de topicalisations ne se justifie guère, sauf lorsqu'il s'agit de passages de discours rapporté ou de commentaire. Le rappel d'un référent cité dans le contexte éloigné, sa réactivation, ne s'effectuent pas d'ordinaire dans une structure topicalisée, mais dans les schémas que nous venons de citer. Le texte argumentatif, en revanche, s'il contient évidemment bon nombre de thématisations, ne peut ignorer les opérations de topicalisation, en particulier quand apparaissent deux mouvements discursifs spécifiques : le développement de plusieurs points d'un raisonnement ou d'un exposé, l'activité de réfutation ; c'est d'ailleurs dans ce dernier cas que l'on peut le mieux percevoir les valeurs "énonciatives" de la topicalisation, ainsi que son rôle de réactivation d'un référent, comme nous le verrons plus loin : il s'agit en effet de rappeler le discours, le point de vue, d'un autre énonciateur, et de prendre position, dans une énonciation marquée comme nouvelle, par rapport à cette proposition rapportée. Il nous a donc semblé intéressant d'examiner ce cas particulier de topicalisation et d'essayer de 
déterminer, dans le processus de formation des marqueurs, et, d'une façon plus large, dans la structuration des énoncés qui contiennent un topique, la place que peut avoir ce schéma dans l'économie générale du système.

4 D'un point de vue diachronique, la période du moyen français ne peut qu'offrir un terrain d'étude particulièrement riche en ce domaine; c'est en effet à cette époque que va se développer la production du texte argumentatif en français, type de texte qui, jusque là, était essentiellement rédigé en latin. Les textes littéraires, les chroniques, adoptent le système caractéristique du texte narratif qui, comme nous l'avons souligné, n'est guère riche en structures topicalisées, la cohérence discursive étant assurée par les progressions thématiques. Des facteurs externes au système de la langue expliquent ce développement de l'emploi du français pour les discours fondés sur la démarche argumentative : traductions d'œuvres philosophiques grecques et latines, abondance des textes polémiques entraînée par la situation politique et les événements de cette période troublée. Il n'est donc pas étonnant que des outils linguistiques spécifiques se forment alors, indispensables à une situation de communication particulière. C'est ainsi qu'apparaît toute une famille de marqueurs destinés à introduire une expression en position de topique, les structures de phrase, la "progression informative" caractéristique du texte narratif ne pouvant suffire à traduire le mouvement énonciatif qui sous-tend une topicalisation. Se pose évidemment la question de l'influence, plus ou moins directe, des textes latins, dans la mesure où il convient de prendre en compte le bilinguisme de la plupart des auteurs : le poids du latin scolastique et de ses structures discursives est sans doute considérable. Ce n'est cependant pas dans la création des marqueurs de topicalisation que cette influence est le plus perceptible; seule la locution quant à peut sans doute être considérée comme un latinisme, et les autres expressions, par exemple celles qui sont formées sur les substantifs regard, égard, ou sur le verbe regarder, apparaissent comme des créations propres au français. En revanche, comme nous allons essayer de le montrer, la structuration de l'énoncé, en particulier la mise en relation de la proposition topicalisée avec le contexte de droite, s'inspire très fortement du modèle latin.

5 En considérant les textes argumentatifs en latin médiéval, dont la plus grande part est constituée par les débats théologiques, eux-mêmes modèles pour les œuvres politiques des $\mathrm{XIV}^{\mathrm{e}}$ et $\mathrm{XV}^{\mathrm{e}}$ siècles, on constate que l'opération de topicalisation s'applique essentiellement à des propositions. Ces textes, qui illustrent la démarche de la « disputatio », sont en effet construits sur un mouvement argumentatif très régulier, dont le principe même est la reprise, la citation d'une proposition, à des fins de réfutation, de discussion. Comme les propositions qui sont ainsi soumises à l'examen critique sont d'ordinaire annoncées dans ce qui constitue une sorte de plan, il est nécessaire de réactiver ces énoncés comme supports d'une nouvelle prédication. Nous sommes alors bien en présence de la configuration typique de la topicalisation, et la question des marqueurs, des outils linguistiques propres à traduire ce type d'organisation, se pose sur deux points principaux: la nature du marqueur, l'expression de l'acte énonciatif qui se développe à partir du constituant ainsi détaché. Un schéma général s'établit, correspondant à : à propos de $\mathrm{P} 1$, je dis que $\mathrm{P} 2$, la proposition $\mathrm{P} 1$ ayant déjà été évoquée dans un contexte plus ou moins immédiat, ou étant supposée appartenir à la connaissance partagée. Les deux paramètres évoqués plus haute, accessibilité du référent et pertinence discursive du choix de ce référent comme support d'une prédication, se retrouvent ici. A cela s'ajoute, d'un point de vue diachronique, un processus de 
grammaticalisation, dans la mesure où le lien syntaxique qui unit les deux parties de l'énoncé peut être très lâche, et que l'on assiste à une structuration progressive de l'énoncé complexe, certains tours paratactiques étant peu à peu remplacés par des relations de plus en plus nettement hiérarchisées. Ce sont ces possibilités que nous allons examiner, en prenant nos exemples dans des textes latins, mais aussi dans des œuvres en français, pour montrer les points de contact entre les deux systèmes discursifs.

Avant de considérer les cas où le déroulement de la réfutation s'effectue à l'intérieur d'une unité qui pourrait être plus ou moins assimilée à une "phrase ", il faut évoquer les tours paratactiques qui répartissent sur plusieurs propositions les différents moments de l'argumentation. La combinaison, dans une structure de topicalisation, du rappel d'une proposition et de la prédication dont elle devient le support n'est pas, en effet, la seule configuration possible dans la démarche de réfutation. L'argumentation se déroule souvent sur deux énoncés successifs, nettement séparés; l'emploi de marqueurs adverbiaux comme vero, autem, permet de souligner le mouvement énonciatif. On remarquera aussi que des verbes de parole ou de jugement indiquent très clairement les deux sources énonciatives :

- Ad hoc dicunt quidam quod [P1]. Nos vero dicimus quod [P2] (Summa Aurea 106)

Sur ce sujet certains disent que (P1). Nous, en revanche, nous disons que (P2)

Il en va de même dans les textes en français, la liaison entre les énoncés s'opérant de plusieurs façons, tout comme l'introduction de P2. L'emploi d'un constituant thématisé renvoyant à la proposition qui précède est relativement fréquent, sous la forme d'une anaphore relative ou démonstrative (à quoi, à cela) :

- Mais on nous dira que [P1]. A quoi nous disons que [P2] (J. de Montreuil, 271)

- on pourroit demander par quel maniere [P1]. A quoi promptement on peut respondre que [P2]

7 Le commentaire que constitue le deuxième énoncé est d'ordinaire construit sur des schémas du type : verbe de parole + que + P2, comme dans les exemples précédents; il peut arriver, plus rarement, que le jugement sur P1 précède l'introduction des arguments de P2, comme dans :

- Ancores veulent maintenir les Angloiz que [P1]. Mais il est tout autrement, car [P2] (M.,

181)

8 Il faut aussi noter que ces tours paratactiques présentent parfois une particularité, en latin comme en français, qui semble annoncer les faits de subordination : un élément, qui peut être un simple substantif, d'ordinaire réponse ou solution, ou toute une proposition, sert de "passage », d'intermédiaire, entre les deux propositions P1 et P2, ce qui permet d'éviter, avant P2, les formules habituelles (à cela on répond que..., etc.) :

et, avec une subordination par que après le substantif :

- Ancores disoient aucuns que [P1]. Response que c'est chose (...) qui ne se peut fonder en

droit (M., 168)

Ce qu'il convient de noter dans l'ensemble de ces tours, c'est que la première proposition ne présente aucune marque de subordination, d'intégration syntaxique qui la mettrait sous la dépendance du contexte de droite.

10 A l'opposé, pourrait-on dire, de ces tours paratactiques, bon nombre d'occurrences de quod + P apparaissent comme parfaitement liées, intégrées à la structure propositionnelle, entrant dans le jeu habituel des faits de rection. Nous n'insisterons pas sur cette possibilité, dans la mesure où il ne s'agit pas de topicalisation; nous noterons simplement que ces constructions témoignent de la généralisation de quod comme marqueur de subordination : 
- Ad hoc debes dicere quod [P] (S., 211)

Sur ce point on dire que $(\mathrm{P})$

- Ad quod est responsio quod [P] (S., 210)

A cela la réponse est que $(\mathrm{P})$

11 L'intégration de quod + P peut aussi se faire en position de sujet, le syntagme verbal principal portant un jugement sur P1, mais on remarquera que l'emploi d'enchâssements conduit parfois à une certaine ambiguïté ; un premier quod peut en effet introduire un verbe de parole correspondant à l'énonciation rapportée, alors qu'une deuxième conjonction introduit le contenu propositionnel. Sous une même disposition linéaire des constituants, il convient donc de voir deux structurations différentes de l'énoncé, en fonction du sémantisme du prédicat principal. Ainsi, dans :

- quod autem obicitur quod [P] non valet (S., 151)

qu'il soit objecté que $(\mathrm{P})$ ne tient pas,

c'est bien l'ensemble initial (quod...P) qui constitue, au plan syntaxique comme au plan sémantique, le sujet de valet; en revanche, dans :

- quod autem dicitur quod [P] falsum est (S., 179)

que l'on dise que (P) (cela) est faux

- quod autem dicitur quod [P] sic est intelligendum (S., 151)

que l'on dise que (P) (cela) doit être ainsi compris,

les prédicats (falsum est, sic est intelligendum) s'appliquent en fait à la deuxième subordonnée (quod P), et non au syntagme complet (quod...quod P) qui pourrait sembler fonctionner comme le sujet; ce qui est faux, ce qui doit être compris de telle ou telle façon, ce n'est pas le fait que $\mathrm{P}$ soit énoncé par certains, mais bien le contenu de la proposition $\mathrm{P}$ enchâssée. Il faut sans doute interpréter ce type d'exemple comme un cas d'« anacoluthe », que l'on peut mettre sur le même plan que les énoncés dans lesquels cette double lecture n'est pas possible, en raison de la nature du verbe principal :

- Quod autem obicitur informitatem tunc esse quam dicit Augustinus (...), dicimus quod ille

intelligit hoc de pura informatione (S., 184)

mais qu'il soit objecté que..., nous disons que...

Nous reviendrons plus loin sur ce type de progression, qui ne correspond pas, nous semble-t-il, à un véritable enchâssement, mais à la juxtaposition de deux «cellules énonciatives » successives.

Les propositions hypothétiques sont parmi les mieux représentées dans la traduction de ce mouvement de réfutation. L'articulation de la protase et de l'apodose permet en effet de présenter l'énonciation rapportée comme une possibilité à laquelle vient s'opposer un argument. Bon nombre d'exemples peuvent laisser penser qu'il y a là des cas indiscutables d'hypotaxe, tant au plan formel, avec la présence de la conjonction si, qu'au plan sémantique, l'enchaînement logique correspondant parfaitement aux relations hypothétiques habituelles :

- Et se l'on me respont : "... ", je dis que [P] (M., 105)

- Et se les Anglois nient [P1], on peut ainsi arguer : [P2]

Il faut cependant s'interroger sur la nature exacte du lien qui unit les deux propositions, la ressemblance avec le français moderne pouvant en fait masquer un fonctionnement différent. A s'en tenir à des exemples tels que ceux que nous venons de citer, aucun indice ne pourrait interdire de voir dans ces énoncés des cas de subordination. Dans d'autres cas, toutefois, l'intégration de la première proposition apparaît comme beaucoup moins nette. On retrouve par exemple le procédé que nous avons déjà relevé, qui consiste à utiliser un substantif (solution, réponse) avant la deuxième proposition, la seule différence avec les cas de parataxe résidant dans l'utilisation de la conjonction si ou d'une locution 
conjonctive équivalente (cf. à ce que, qui traduit le si latin dans le deuxième exemple cidessous) :

- Et si forsan diceretur [P1], solutio quod [P2] (S., 227)

et s'il arrive qu'on dise (P1), solution que (P2)

- Et d'autre part, à ce que aucuns Anglois opposent que [P1]. Solution : que [P2] (M., 172)

- Et se ilz vouloient faire un autre argument de Semiramis, que [P1], response que [P2] (M.,

173)

Ce type de construction, que l'on pourrait considérer comme intermédiaire entre la parataxe et les cas plus nets de subordination, ne se limite pas aux tours hypothétiques ; il apparaît aussi avec les formes où, ubi :

- Et ou les Anglois alleguent que [P1], response que [P2] (M., 173)

- Ubi autem alii argunt: [P1]. Soluitur argumentum, quod [P2] (M., 230)

- Là où d'autres allèguent (P1). L'argument ne tient pas, car (P2)

Même s'il est difficile de tirer des conclusions d'un système de ponctuation relativement hétérogène, on peut interpréter, nous semble-t-il, la séparation des deux propositions comme la succession de deux mouvements énonciatifs; la première proposition correspond à une annonce, à une sorte de "titre ", plus ou moins lié au commentaire qui le suit, pouvant être comparé, toutes proportions gardées, au fonctionnement des groupes introduits par la préposition de du latin en tête de rubrique, sans liaison syntaxique forte avec le contexte de droite qui constitue le développement. Cette relation lâche, qui n'est pas encore du domaine de l'hypotaxe et qui pourrait être rapprochée, pour la même période, des emplois de quant à, se rencontre aussi fréquemment avec la conjonction ut (pour). Ici encore, tout se passe comme si une ellipse du verbe de parole mettait directement en contact topique et commentaire :

- Et ut respondeatur illis qui aiunt quod [P1], istud sustineri non potest (M., 228)

- Et pour respondre à ceux que aucuns ont argué, que [P1] : c'est chose qui ne se peut soustenir

$$
\text { (M., 275) }
$$

- Et ut fiat satis quibusdam, qui [P1]. Respondetur talia dicentibus [P2] (M., 318)

- Et pour respondre à aucuns qui disoient que [P1]. En quoi il n'a point d'aparence (...), vu que [P2]

$$
\text { (M., 321) }
$$

On peut s'attendre à ce que se forment, à partir de telles constructions, des séquences dans lesquelles une évolution vers la phrase complexe semble se produire, mais où se maintient en fait le phénomène d'ellipse, comme dans l'exemple suivant, où le contenu de P2 n'est en rien conditionné par celui de P1 :

- Et s'il dit que sa mere lui donna et transporta tel droit (...), il est evident que trop mieux

madite dame d'Orleans le peust donner et transporter au roi Philippe (M., 170),

exemple dans lequel l'explicitation de la liaison logique conduirait à : s'il dit..., on répond qu'il est évident que...

Notons enfin que ces faits d'ellipse ne s'accompagnent pas toujours de marqueurs comme si, pour, où, qui traduisent un certain degré de dépendance ou, du moins, une relation sémantique; seule demeure la conjonction quod, à laquelle correspond ce que, plus fréquemment que le simple que, mais rien ne signale une quelconque fonction de cette proposition par rapport à ce qui serait une proposition enchâssante :

- Quod autem obicitur quod [P1], dicimus quod [P2] (S., 184) qu'il soit objecté que..., nous disons que...

- Quod autem sic citatur Scriptura (...), habes per simile in alia translatione...(S.,199) que l'on cite ainsi l'Ecriture..., on a la même chose dans une autre traduction

17 Nous évoquerons à présent les cas où la topicalisation ne s'effectue pas sur une structure propositionnelle introduite par quod, mais sur un élément à valeur nominale; 
remarquons toutefois que ce schéma permet souvent une démarche identique à celle que nous venons d'illustrer, dans la mesure où l'élément nominal peut être lui-même le support d'une prédication, comme dans :

- Ad sextum quod queritur, scilicet [P], habes solutionem (...) (S., 187)

- quant au sixième point qui est soulevé, à savoir [P], on a la solution...

L'emploi fréquent de la préposition ad favorise d'ailleurs le groupe hoc quod, qui a pour équivalent le français ce que :

- Ad hoc autem quod obicitur quod [P1], concedimus quod [P2]

quant au fait que l'on objecte que [P1], nous concédons que [P2]

La présence de P1 n'est évidemment pas obligatoire :

- Ad secundum objectum dicimus quod [P]

sur le second point nous disons que

19 Ce qui est intéressant dans ces constructions, c'est la relation systématique qui s'établit, dans ce type d'exemple, entre l'opération de topicalisation et l'occurrence d'un verbe de parole ou, plus largement, d'un verbe renvoyant à la démarche argumentative. On peut considérer que le choix de la préposition ad est, en quelque sorte, motivé par le sémantisme du verbe, même s'il n'y a pas toujours rection à proprement parler. Il en va de même avec la préposition de, qui survient, comme on peut s'y attendre, en corrélation avec le verbe dicere :

- De prima (= causa) dicimus quod ipsa est falsa (S., 168)

de la première cause nous disons qu'elle est fausse

- De illo autem qui noluit obedire (...), dicimus quod (...) meruerat (S., 162)

mais de celui qui n'a pas voulu obéir, nous disons qu'il a mérité...

Mais on peut aussi remarquer la même tendance avec un introducteur comme circa, qui est se trouve d'ordinaire accompagné d'une forme verbale ouvrant la partie de commentaire :

- Circa primum videtur quod non (S., 289)

- Circa secundum articulum queritur an (...) (S., 291)

- Circa primum duplex est opinio (S., 286)

21 Sans vouloir établir un lien chronologique entre les deux types de topicalisation (le topique correspondant à une proposition ou à un constituant nominal), on pourra relever cette propriété commune qui consiste à traduire le mouvement énonciatif qui introduit le commentaire ; c'est en quelque sorte la caractéristique essentielle de la topicalisation qui se trouve ainsi explicitée.

Pour examiner ce type de topicalisation dans des textes en français, nous nous appuierons à présent sur un type particulier de document, la correspondance, qui, tout en ne développant pas le même type d'argumentation scolastique, peut sans doute être influencé par le système latin que nous venons de décrire. Considérons par exemple le corpus des lettres de Louis XI, qui couvre la période de 1444 à 1483. Les topicalisations y sont nombreuses, avec une grande fréquence du marqueur au regard de, mieux représenté que quant à et touchant. Ces locutions introduisent essentiellement des groupes nominaux, comme dans :

- Mais au regard de la guerre sur mer, elle est demeurée en l'état que (...) (108)

- Quant à notre venue par deçà, nous vous en avons naguère écrit (126)

- Touchant sa pension, je lui ai fait appointer (...) (300)

Les constituants ainsi topicalisés renvoient souvent, comme on peut s'y attendre dans un échange de lettres, à des référents préalablement évoqués par les correspondants. Face à cette abondance naturelle de syntagmes nominaux, la topicalisation d'une proposition 
apparaît bien plus rarement. Lorsqu'elle survient, c'est d'ordinaire la locution au regard de ce que qui se trouve utilisée, mais on peut relever aussi quelques occurrences de à ce que et de quant à ce que. Il faut noter une propriété intéressante dans l'organisation générale de l'énoncé, propriété qui ne semble pas souffrir d'exception: le marqueur (au regard de ce que) introduit un verbe de parole qui fait référence à l'acte énonciatif auquel va répondre le commentaire. L'exemple suivant fait bien apparaître ce type d'enchaînement :

- Et au regard de ce que me faites savoir que Philippe de Savoie veut aller en Piémont, et qu'il y pourrait avoir danger, il n'en y a point, car (...) (103)

Nous retrouvons ici, toutes proportions gardées, le mouvement caractéristique des textes argumentatifs déjà évoqués plus haut : la topicalisation consiste à reprendre l'énonciation d'un interlocuteur et à bâtir, à partir de ce rappel, une nouvelle prédication, portée par le commentaire. Ce qui doit être souligné, c'est la dissymétrie opérée par l'ellipse du verbe de parole qui pourrait introduire le commentaire; là où l'on attendrait : au regard de ce que vous dites que ( $(\mathrm{P} 1)$, je réponds que (P2), seule la partie topicalisée renvoie explicitement à l'acte de parole. On pourrait par ailleurs s'attendre à ce que P1 suive immédiatement le marqueur, ce qui correspondrait aux topicalisations de syntagmes nominaux (au regard de ce que Philippe veut aller en Piémont, ...), mais ce schéma n'est pas attesté dans le corpus et on peut sans doute voir dans cette systématisation le résultat de la situation quasiment constante de discours rapporté et de démarche argumentative. Un passage comme :

- car ce que le Prince a été à Saint-Omer, ce a été pour gagner Monseigneur de Saint-André (426),

s'il relève bien du discours rapporté, renvoie à un fait attesté, non prétendu, qui n'est pas soumis à discussion; d'où l'emploi de ce que (et non de : au regard de ce que vous dites que) et l'absence de verbe de parole. Ces textes de moyen français semblent donc faire aller de pair l'emploi des marqueurs devant une structure propositionnelle et le rapport d'une énonciation, même si l'enchaînement sur le commentaire se fait par rapport au contenu de P1. Ainsi, dans l'exemple cité plus haut, le commentaire (il n'y a point de danger) répond-il à la proposition qui précède (il pourrait y avoir danger), indépendamment, pourrait-on dire, de la formule introductrice (au regard de ce que vous dites que). Les extraits suivants illustrent ce mouvement énonciatif, qui conduit du rappel d'une énonciation à un enchaînement sur le contenu propositionnel :

- Quant à ce que vous dites que je m'avance et ne me laisse point amuser de paroles : je dois

demain ouïr des nouvelles de Bretagne (...) (237)

- Et au regard de ce que le pape dit que j'ai été un peu ému, mais que je serai tantôt rapaisé, je ne suis pas si aisé à émouvoir qu'il dit (405)

L'énonciation qui sous-tend la partie de commentaire peut être explicitée dans l'emploi de formes à l'impératif :

- Et au regard de ce que vous dites que le seigneur Boffille ne se veut consentir que (...), faites-le ainsi qu'il avisera (290)

- Au regard de ce que vous m'écrivez que vous l'avez accordé pour ne pas mettre les choses en rupture, ne leur accordez rien pour peur de rupture (422)

Il faut aussi noter une ambiguïté, qui n'est pas propre aux tours topicalisés, mais provient du fonctionnement de ce que; ce groupe peut en effet être parfois interprété comme un relatif, la conjonction que qui suit le verbe "développant ", en quelque sorte, ce pronom objet, ce qui pourrait se paraphraser par : quant à ce que vous dites, à savoir que (...). Dans l'exemple suivant, l'intercalation du syntagme au sujet de $X$ entraîne assez facilement cette lecture :

- A ce que vous nous écrivez des deux cousins, lesdits Niklaus et Guillaume de Diesbach, que nous veuillions les avoir en notre spéciale recommandation, nous les aurons encore plus, puisque (...) (181) 

évidemment la seule possible et aucune ambiguïté ne survient, comme dans :

- Et au regard de ce que le seigneur de Baudricourt vous a dit de par nous, tenez vous sûrs que nous avons vous et ses pays en singulière recommandation (272)

- Et au regard de ce que vous baillerez, je vous en baillerai votre acquit (458)

On rejoint ici la topicalisation des syntagmes nominaux, qui renvoient à un référent non propositionnel ; la différence entre les deux constructions explique sans doute le fait que la « reprise » dans le commentaire par une forme anaphorique soit plus fréquente dans ce dernier cas. Lorsque le topique est une proposition, le rappel d'un référent est évidemment possible, mais ce cas est tout de même marginal.

Ces structures en: préposition $+c e$ que $+\mathrm{P}$ sont, comme on peut s'y attendre, peu intégrées au système des relations interphrastiques; dans bon nombre d'occurrences, elles apparaissent comme des sortes de «titres », d'annonces, en vue d'un développement plus ou moins long, le commentaire dépassant les limites de la proposition ou de ce qui correspondrait approximativement à la phrase moderne. Cette " portée » large de ce type de topicalisation est bien illustrée par le passage suivant où l'on peut identifier deux topicalisations successives :

Monseigneur du Plessis, j'ai reçu vos lettres et, au regard de ce que monseigneur d'Arguel vous a répondu qu'il sera prêt de s'en venir vers moi et de me servir, mais que (=pourvu que) je lui aie fait délivrer son père et appointer sa pension: Monseigneur du Plessis, que je délivre son père, je n'en ferai rien (...) Touchant sa pension, je la lui ai fait appointer (...) (300)

Le premier topique annonce les deux points qui seront développés dans la lettre (la délivrance du père, la pension) et on ne peut rattacher syntaxiquement le constituant introduit par au regard de au contexte de droite. Nous retrouvons ici les caractéristiques illustrées plus haut par les extraits de Jean de Montreuil, qui comportaient un terme comme réponse, intermédiaire entre les deux composantes de l'énoncé. A partir de ce premier topique, se succèdent les deux points annoncés, sous la forme de deux syntagmes topicalisés, le rappel s'effectuant par la simple conjonction que (que je délivre son père) : il ne s'agit plus ici de rapporter une énonciation qui servirait de point de départ au commentaire - cette opération correspond à la structure qui ouvre la lettre (au regard de ce que) -, mais simplement de "reprendre ", comme on le ferait pour des thèmes dérivés, chacun des référents. On notera que, dans cette deuxième opération de topicalisation, le topique que je délivre son père est rappelé par l'anaphore en, de même que le groupe sa pension est repris par le pronom la dans la deuxième partie du commentaire.

Il est intéressant de relever, dans un autre texte, un exemple de progression identique, mais contenant des syntagmes nominaux topicalisés et non des propositions :

- Au regard de l'argent que vous demandez, ce que j'ai, je le baillerai volontiers, et, ce que je

$n^{\prime}$ 'aurai point, j'en ferai volontiers finance à Paris (...) (Le Clerc, 1502)

Sans qu'il y ait liaison chronologique "directe", succession, entre les deux types de topicalisations (les enchaînements diachroniques pourraient faire l'objet d'une étude particulière), on peut retrouver des traces, dans les tours avec groupes nominaux, de ce mouvement énonciatif que nous venons d'observer. En s'en tenant au cas de la locution au regard de, on constatera, par exemple, que le référent topicalisé n'est d'ordinaire pas immédiatement repris dans le commentaire; un verbe de parole ou une expression contenant une modalisation jouent en quelque sorte un rôle d'intermédiaire entre le topique et le contexte de droite. Sur le modèle général : au regard de $X$, je peux dire que $P$, l'énoncé conserve ainsi une marque qui rappelle qu'un acte de parole spécifique 
accompagne la deuxième partie de cette opération de topicalisation. Les exemples suivants présentent ce type de progression :

- Au regard des prévosts à ferme, on sait bien qu'il faut qu'ils vivent et payent leur ferme (Juvénal des Ursins, 1445)

- Au regard des premiers, il n'est aucun doute que on ne les doie appeler au Conseil (id.)

- Au regard de Gervaise, il me semble qu'il doit ordonner de la ville ceux qui (...) (Jean de Bueil, 1461)

- Au regard de l'âme, nous devons savoir que qui se garde de pécher mortellement qu'il est sauvé (A. de La Sale, 1456)

Il conviendrait évidemment, dans une approche plus générale, d'analyser les relations qu'entretiennent ces tours, ne serait-ce que du point de vue chronologique, avec les énoncés qui ne présentent pas ces éléments intermédiaires et dans lesquelles le topique se trouve immédiatement mentionné, sous la forme d'une anaphore, dans la structure propositionnelle que constitue le commentaire. Cette possibilité est déjà bien attestée à l'époque qui nous intéresse ici, comme dans les énoncés suivants, dont le modèle ne cessera d'être productif dans l'histoire du français :

- Au regard des autres, ils sont bonnes gens et hardis (Jean de Bueil, 1461)

- Au regard des draps de laine, il y en avoit en grande quantité (André de La Vigne, 1495)

A ce stade de développement, ce schéma se confond, dans une certaine mesure, avec celui des topicalisations qui correspondent à la phrase "segmentée ", qui ne comporte pas d'expression introductrice de topique, mais qui présente aussi la reprise anaphorique du syntagme initial. Les structures propres au texte argumentatif pourraient ainsi être considérées comme une des sources du processus de grammaticalisation des constructions topicalisées du français. Ce rôle du niveau discursif est particulièrement intéressant à observer, car il permet de comprendre comment l'opération de topicalisation se trouve liée, de façon plus ou moins explicite, au domaine énonciatif.

\section{BIBLIOGRAPHIE}

\section{Textes cités}

Guillaume d'Auxerre, Summa Aurea, Liber Secundus, Tomus I, éd. J. Ribaillier, CNRS, 1982.

Jean de Montreuil, Opera, volume II, L'oeuvre historique et polémique, éd. N. Grévy, E., Ornato, G. Ouy, Turin, G. Giappichelli, 1975.

Louis XI, Lettres, éd. H. Dubois, LGF, 1996.

\section{Bibliographie}

COMBETTES, B. (1988) « Typologie textuelle et structures syntaxiques : le texte argumentatif en moyen français ", in U. Jokinen et P. Sihvonen (eds), Approches du moyen français, 12-28, Univ. de Jyväskylä.

COMBETTES, B. (1992) «Contraintes syntaxiques et valeurs discursives : l'opposition que / ce que en moyen français ", in E. Sakari et H. Häyrynen (eds), Approches du moyen français II, 17-30, Univ. de Jyväskylä. 
DOHERTY, M. (2001) « Cleft-like sentences », Linguistics, 39, 607-638.

DOHERTY, M. (2001) « Discourse functions and language- specific conditions for the use of cleft(like) sentences : a prelude », Linguistics, 39, 457-462.

HAIMAN, J. (1978) « Conditionals are topics », Language, 54, 564-589.

JACOBS, J. (2001) «The dimensions of topic-comment », Linguistics, 39, 641-681.

LAMBRECHT, K. (1994) Information Structure and Sentence Form. Topic, Focus, and the Mental Representation of Discourse Referents, Cambridge: Cambridge University Press.

LAMBRECHT, K. (2001) « A framework for the analysis of cleft constructions », Linguistics, 39, 463516.

RYCHNER, J. (1970) L'articulation des phrases narratives dans la Mort Artu, Genève : Droz.

\section{RÉSUMÉS}

En examinant, dans des corpus de textes argumentatifs en latin médiéval et en moyen français, le cas des propositions topicalisées, on analyse le processus de grammaticalisation qui conduit de la parataxe à des constructions dans lesquelles le constituant initial se trouve intégré dans un énoncé qui se rapproche ainsi de la phrase complexe. L'activité de réfutation et, plus largement, celle de commentaire, apparaissent comme particulièrement propres à favoriser ce genre d'évolution. Les marques énonciatives, sous-jacentes à toute opération de topicalisation, se trouvent justifiées par la présence du discours rapporté contenu dans le topique et par l'acte de langage inhérent au commentaire.

\section{AUTEUR}

\section{BERNARD COMBETTES}

Université Nancy 2 et UMR-ATILF 